\title{
Advances in Intelligent Tutoring Systems: Problem-solving Modes and Model of Hints
}

\begin{abstract}
Alla Anohina
Abstract: The paper focuses on the issues of providing an adaptive support for learners in intelligent tutoring systems when learners solve practical problems. The results of the analysis of support policies of learners in the existing intelligent tutoring systems are given and the revealed problems are emphasized. The concept and the architectural parts of an intelligent tutoring system are defined. The approach which provides greater adaptive abilities of systems of such kind offering two modes of problem-solving and using a two-layer model of hints is described. It is being implemented in the intelligent tutoring system for the Minimax algorithm at present. In accordance with the proposed approach the learner solves problems in the mode which is the most appropriate for him/her and receives the most suitable hint.
\end{abstract}

Keywords: intelligent tutoring system, problem-solving mode, hint

\section{Introduction}

Emerging of a knowledge society and growing demands for highly skilled and educated labor force claim for changing traditional teaching and learning processes. One way of changes is related with an integration of various kinds of computer-based learning systems as supplements to conventional teaching methods. However, it is necessary to provide intelligent and adaptive abilities of a software system in order it could take over a role of a teacher in effective way. This idea is not new one as it is exploited in intelligent tutoring systems for more than 30 years since the earliest SCHOLAR system [1] appeared. During this time a huge amount of intelligent tutoring systems has been implemented for different areas, for example, for mathematics, physics, medicine, informatics and computer science $[2,3,4,5,6,7,8,9$, 10]. Nowadays developments of systems of such kind have received new breath with the appearance of the agent paradigm [11].

However, adaptive abilities of intelligent tutoring systems still are not high enough, particularly regarding modes of practical problems solving and support of a learner in this process. Solving of domain problems is an important part of intelligent tutoring systems, as it allows to deepen the acquired theoretical knowledge in practice, but the mere solving is unlikely to lead to improved skills or deeper understanding of a subject matter. Learning often takes place best when the learner receives feedback from the system. Feedback is a way to improve the learning process on the basis of continuous assessment of learning results, the analysis of their quality and performance of necessary corrections. Feedback encourages desired learning behavior and discourages undesired one, allows to understand how successfully the learner acts, whether he/she applies relevant knowledge, and it provides opportunities to correct misconceptions.

In case of intelligent tutoring systems feedback is the various reactions of the system to learner's learning behaviour. In its turn, a hint is only one form of feedback. Unfortunately, little prior researches have been done which are devoted to the general issues of hints formation in intelligent tutoring systems. The most significant work is [12], containing the description of the results of studying hints used by experienced tutors and an attempt to formulate a strategy for using hints in intelligent tutoring systems. According to [12] hints encourage the student to engage in active cognitive processes that are thought to promote deeper understanding and long-term retention.

As it is pointed in [13], the developed intelligent tutoring systems have relatively simple and inflexible hinting policies, which more often demand from the learner to follow a prescribed problem-solving strategy and, therefore, hints are always aimed at the next step which should be taken accordingly to the 
strategy. The authors draw attention to two problems: inflexible choice of the steps targeted by hints and proceeding of hints from the most general to the most specific.

The analysis of the existing intelligent tutoring systems allows to make the following conclusions about reactions of a system to actions of a learner. Typically the system gives the learner an immediate feedback after each performed action or step during problem-solving irrespective of the fact whether the action or the step was correct or incorrect. Such policy prevents the learner from proceeding along a wrong solution path. The examples of immediate feedback are found in $[5,8,9,14,15]$. But may be a learner would like to make a series of steps and after that to receive feedback about correctness and to find by his/herself what step has led to the incorrect solution?

The system usually provides a special button or tool, which the learner can use to request a hint. In AlgeBrain [5] such tool is an animated agent. The system responds with two types of support: generalized "Here's what I'm expecting you to do at this point" help text and a hint specific to the current state of the problem. In Andes [9] there are two buttons. One of them gives help "what's wrong with that?" on an incorrect entry. Other button provides a hint about the next step in problem-solving.

Typically hints are organized in a range from the most general to the most specific. The general hint as a rule contains a minimum information on an error. Further the informativeness of hints increases. The most specific hint clearly specifies or shows what should be done. Hints are given sequentially. There is a number of systems which use this approach, for example, $[5,8,10,14,16,17]$. The organization of hints from the most general to the most specific is not flexible enough. The insufficient amount of information in a hint can cause frustration and desire to request the subsequent hints without attempts to solve a problem by the learner. Information, which specifies necessary actions after first request of a hint, in its turn, is contradictory to the learning process. Thus, mechanisms, which will allow to implement individual system's reactions for each learner giving such amount of information, which will help and at the same time will provide certain cognitive load, are necessary. The example of adaptive hinting is described in [18]. The authors use learner's proficiencies to select an appropriate hint. The learner with high proficiency at a particular skill receives the more subtle hint. The less proficient learner is presented with a more obvious hint. The authors point out that this is better than require learners to wade through several levels of hints before they receive material that is appropriate to their knowledge level.

The paper describes an approach which provides greater adaptive abilities of intelligent tutoring systems supporting two modes of problem-solving and using a two-layer model of hints. Thus, the learner solves problems in the mode which is the most appropriate for him/her and receives the most suitable hint. The aforementioned approach is being implemented in the intelligent tutoring system for the Minimax algorithm at present.

The paper is organized as follows. Section 2 defines the concept and the architectural parts of an intelligent tutoring system. The developed approach based on two modes of problem-solving and a twolayer model of hints is discussed in Section 3. Section 4 describes the intelligent tutoring system for the Minimax algorithm in which the proposed approach is being implemented. Finally, conclusions are presented, and some directions for future work are outlined.

\section{Intelligent tutoring systems}

Despite of a broad variety of the developed systems an unequivocal and exhaustive definition of an intelligent tutoring system still does not exist. However, it is possible to list the most often mentioned characteristics of systems of such kind [10, 19, 20, 21, 22, 23, 24]. Thus, the intelligent tutoring system is a computer-based system. It is an intelligent system because it uses principles and methods of artificial intelligence [25] such as knowledge representation, inference mechanisms and machine learning in its structure and operation. An intelligent tutoring system is an adaptive system as it alters aspects of its structure, functionality or interface for the particular user and his/her changing needs over time [26]. It 
emulates a human teacher, tries to provide benefits of individual (one-on-one) tutoring, and is based on the theory of learning and cognition.

Furthermore, intelligent tutoring systems are characterized by the fact that they store three basic kinds of knowledge [20, 27]: domain knowledge, knowledge about learners, and pedagogical knowledge. The knowledge types determine three main parts of the system's architecture: the domain knowledge, the student diagnosis module, and the pedagogical module. An intelligent tutoring system, as any other software intensively communicating with users, needs a part of the architecture responsible for the interaction between the system and the learner. It is a communication module or interface which controls screen layouts, interaction tools, etc. However, each system can contain additional components the presence of which depends on the following factors: features of problem domain, locking down of separate functions of the basic constituent parts in the isolated components of the structure, technology used for system implementation, and additional functional capabilities of the system. The general architecture of an intelligent tutoring system is shown in Figure 1.

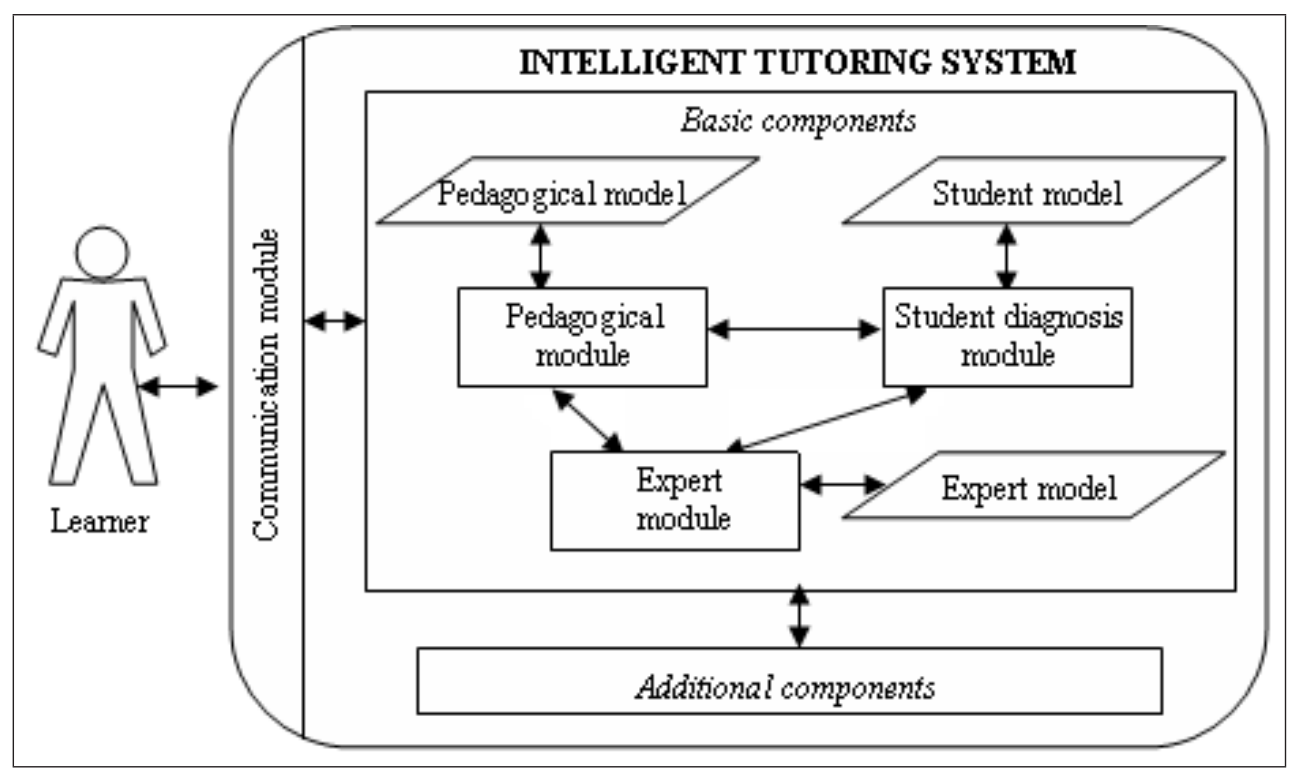

Figure 1: The general architecture of an intelligent tutoring system (adopted from [28])

The domain knowledge is the knowledge the system is teaching. Most often it is incorporated in the expert model which represents skills and expertise that an expert in a particular domain holds. The model serves as a standard for evaluating the learner's performance and the knowledge level. The domain knowledge can include fragments of theoretical materials, texts of practical tasks and attributes related with them, explanatory units, rules and principles used in the domain, etc. Typically, it is represented within the system using logical, procedural, network or structured knowledge representation schemes [29]. Moreover, the domain knowledge is organized in a certain way, commonly, as a hierarchy, for example, a topic includes some units, which consist of several chapters. The expert module generates solutions of problems for their further comparison with solutions of the learner.

The student diagnosis module carries out the student diagnosis process that collects information about the learner, analyzes it and stores in the student model. The student model is formed for a particular learner and serves as an input to the pedagogical module which tailors the learning process to the needs of the learner. The model contains learner's identifying information, information on the current knowledge level of the learner, information about learner's cognitive, emotional and psychological features, his/her past experience, interests, and system's options usage by the learner.

The pedagogical module provides a knowledge infrastructure for adaptation of the learning process to characteristics and needs of a learner without interventions of a human-teacher. It implements the 
learning process on the basis of teaching strategies and instructions held in the pedagogical model. The primary tasks of this module are selection and sequencing of learning material that is the most suitable for the learner, determining of the type and content of feedback and help, and answering questions from the learner.

\section{The proposed approach}

\subsection{The problem-solving modes}

Generally, there are two possibilities regarding moments of feedback delivering: an immediate feedback after each step or action in problem-solving and feedback after submission of a whole solution to the problem. It is a basis for two modes of problem-solving in the proposed approach. In the completeness mode a learner chooses the moments of feedback presentation to check correctness of a series of steps. So, he/she can perform one or more steps solving a problem and then to require checking of the performed steps. The system provides feedback about correctness of his/her previous actions and the learner by his/herself should determine what step has led to the incorrect solution. This mode is similar to reinforcement learning [30] which is widely used in artificial intelligence. In the step-by-step mode the system monitors each problem-solving step and gives feedback about its correctness.

There are four variations of the step-by-step mode regarding a kind of information given to the learner:

- The learner receives both positive and negative feedback. In the case when the learner has performed the correct action he/she is praised (receives a positive feedback, or a reward). If the step was incorrect, criticism (negative feedback) is given to the learner. Moreover, negative feedback can be given in two different forms: only as a text, which informs that the action was incorrect, and as a text about the incorrect step together with a hint about how to improve his/her operation.

- The learner receives only negative feedback. In this case negative feedback also can be given in two different ways described above.

In the completeness mode the learner is not praised or criticized for each performed step. Instead of it he/she receives a total estimation of all performed actions. The estimation specifies how far the learner is from his/her goal: the correct solution of a problem. The total estimation can have a positive or negative deviation regarding difference between a number of correctly and incorrectly performed steps. In case when the mentioned difference exceeds some admissible value, a hint can be given to the learner. Thus, there are two variations of the completeness mode regarding a kind of feedback:

- The learner receives only a total estimation of the performed steps.

- The learner receives a total estimation of the performed steps together with a hint about how to improve his/her operation.

It is obvious, that it is necessary to provide an opportunity to change the problem-solving mode and a kind of feedback by the learner, as well as, to request a hint in case when he/she receives only the text of feedback. Thus, the general scheme of the problem-solving modes and kinds of feedback is displayed in Figure 2.

It is necessary to stress, that the described problem-solving modes can be implemented only if the process of finding of a problem solution consists of several (homogeneous or heterogeneous) steps. 


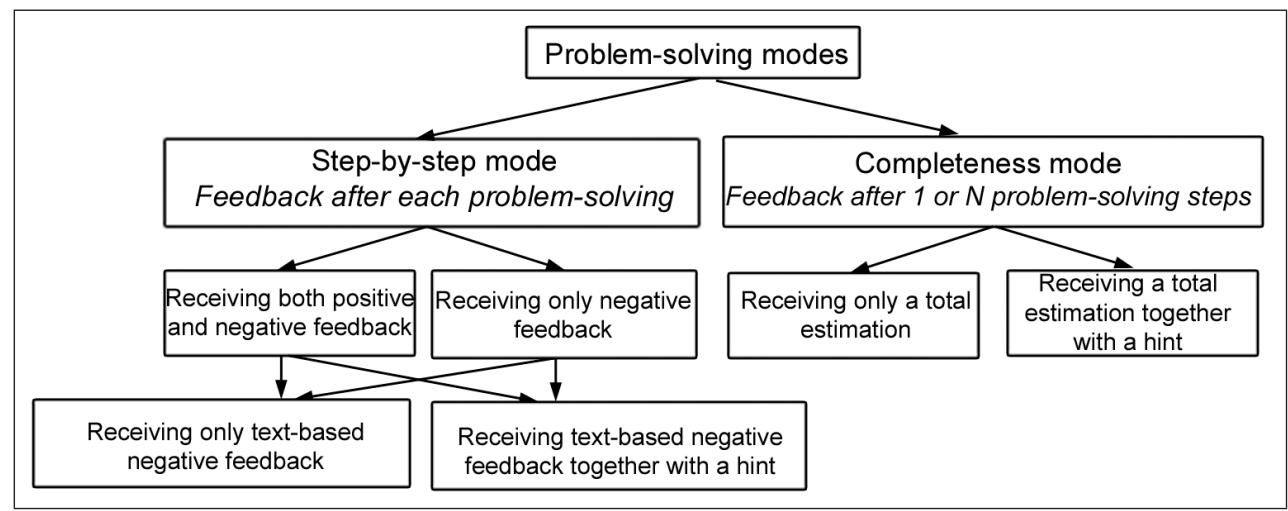

Figure 2: The problem-solving modes and kinds of feedback for an intelligent tutoring system

\subsection{The model of hints}

The model of hints in the proposed approach defines two layers (Figure 3): a layer of the general hint categories and a layer of hints within the general categories. There are three general hint categories: general hints, hints of average informativeness, and specific hints. Each category contains one or more hint, which also are ranged from less informative to more informative.

The model allows the learner to receive a hint that is the most suitable for him/her. Before the learner starts to work in problem-solving mode testing should be taken with the purpose to determine a general hint category which is suitable for the learner. Further requesting help during problem-solving the learner will receive an average by number hint from the hint category suitable for him/her. If after receiving of a hint the learner is not capable to execute a correct action, he/she is presented with a subsequent hint. The process proceeds while he/she will not reach last hint for the given error. Such approach spares the learner from being presented with informativeless hints. Contrary, the learner timely receives a hint providing help and certain cognitive load, therefore, reducing an opportunity of frustration, floundering and loss of interest to learning.

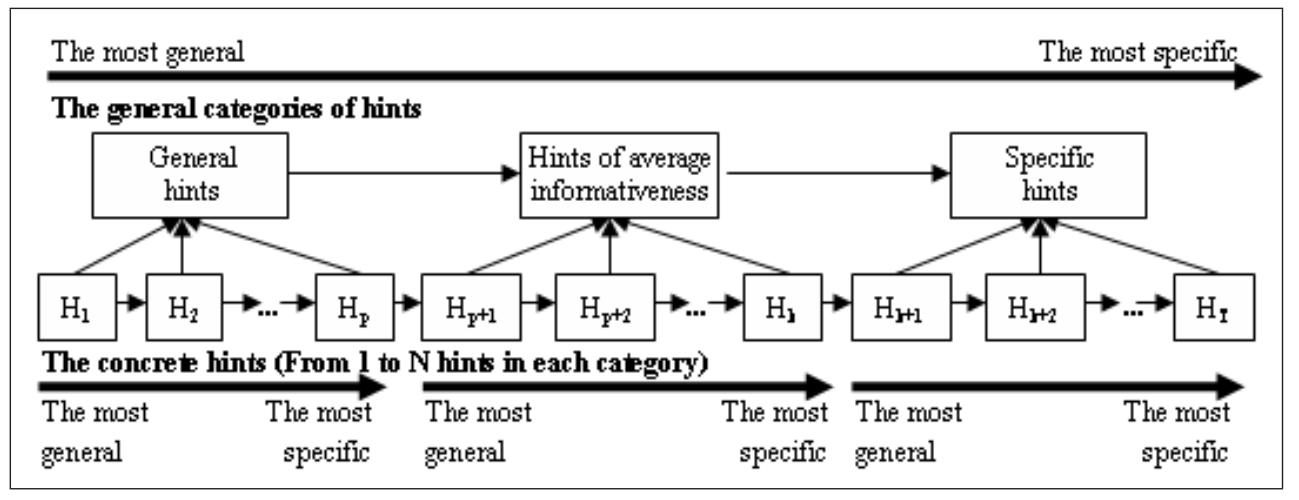

Figure 3: The two-layer model of hints (adopted from [28])

\section{The intelligent tutoring system for the Minimax algorithm}

The described approach is being implemented in the intelligent tutoring system for a topic of the learning course "Fundamentals of artificial intelligence" at the faculty of Computer Science and Information Technology at Riga Technical University. The topic is related with the algorithm for implementing 
two-person games with full information, i.e., the Minimax algorithm [29] which example is given in [31] in details.

All practical problems directed to the development of skills of applying of the Minimax algorithm consist of a sequence of homogeneous steps. Let's consider one of them. A game tree is presented to the learner. It has arcs which are inadmissible for a game with full information: arcs between nodes at the same level or through levels. As a rule, there are from 3 up to 5 wrong arcs in the tree. The learner should find them and to remove from the tree. Thus, a removal of one (wrong or correct) arc is a step in this task. It is obvious, that tasks for the Minimax algorithm are a fine example of an opportunity to provide two problem-solving modes in the system.

At present the following tasks regarding the developed approach are completed: possible mistakes and hints corresponding to them are defined for each problem, sets of hints for each mistake are divided into categories according to the two-layer model of hints described in the previous section, and the user interface of both problem-solving modes is developed.

The architecture of the system corresponds to the general architecture of an intelligent tutoring systems displayed in Figure 1. The system carries out assessment of an initial learner's knowledge level on the topic, provides the theoretical knowledge acquiring mode and the practical problem-solving mode with preliminary determining of the problem-solving mode and a category of hints most suitable for the learner, and gives a final assessment of the achieved knowledge level.

\section{Conclusions and future work}

Adaptive abilities of intelligent tutoring systems are not high enough especially regarding problemsolving modes offered to a learner and ordering of hints from the most general to the most specific. The paper presents an approach which allows the learner to work in the problem-solving mode that is the most appropriate for him/her and to receive the most suitable hint. The proposed two-layer model of hints can reduce frustration, floundering and loss of interest to learning that are inevitable in case when the learner receives too little support during problem-solving.

At present, the proposed approach is in a stage of development. Firstly, it is necessary to determine, how testing for the problem-solving mode and a category of hints most suitable for the learner may be implemented. Secondly, both psychological and pedagogical foundations of the proposed approach should be specified. After the implementation of the approach in the intelligent tutoring system for the Minimax algorithm the testing of the system will be done in the corresponding learning course together with questioning of learners and the subsequent interpretation of the received results.

\section{References}

[1] J. R. Carbonell, AI in CAI: An Artificial Intelligence Approach to Computer-Assisted Instruction, IEEE Transactions on Man-Machine Systems, Vol. 11, No. 4, pp. 190-202, 1970.

[2] J. S. Brown and R. R. Burton, A Paradigmatic Example of an Artificially Intelligent Instructional System, International Journal of Man-Machine Studies, Vol. 10, pp. 323-339, 1978.

[3] J. S. Brown, R. R. Burton and J. de Kleer, Pedagogical, Natural language, and Knowledge Engineering Techniques in SOPHIE I, II and III, In D.H. Sleeman and J.S. Brown (Eds): Intelligent Tutoring Systems, Academic Press, London, 1982.

[4] J. R. Anderson and B. J. Reiser, The Lisp Tutor, Byte Magazine, Vol. 10, pp. 159-175, 1985.

[5] S. R. Alpert, M. K. Singley and P. G. Fairweather, Deploying Intelligent Tutors on the Web: an Architecture and an Example, International Journal of Artificial Intelligence in Education, Vol. 10, No. 2, pp. 183-197, 1999. 
[6] V. Devedzic, J. Debenham and D. Popovic, Teaching Formal Languages by an Intelligent Tutoring System, Educational Technology \& Society, Vol. 3, No. 2, pp. 36-49, 2000.

[7] M. Hospers, E. Kroezen, A. Nijholt, R. den Akker and D. Heylen, An Agent-based Intelligent Tutoring System for Nurse Education, In J. Nealon and A. Moreno (Eds): Applications of Intelligent Agents in Health Care, Birkhauser Publishing Ltd, Basel, Switzerland, 2003.

[8] N. Matsuda and K. VanLehn, Advanced Geometry Tutor: an Intelligent Tutor that Teaches Proof-Writing with Construction, Proceedings of the 12th International Conference on Artificial Iintelligence in Education, Amsterdam, pp. 443-450, 2005.

[9] K. VanLehn, C. Lynch, K. Schulze, J. A. Shapiro, R. Shelby, L. Taylor, D. Treacy, A. Weinstein and M. Wintersgill, The Andes Physics Tutoring System: Lessons Learned, International Journal of Artificial Intelligence in Education, Vol. 15, No. 3, pp. 147-204, 2005.

[10] R. S. Crowley and O. Medvedeva, An Intelligent Tutoring System for Visual Classification Problem Solving, Artificial Intelligence in Medicine, Vol. 36, No. 1, pp. 85-117, 2006.

[11] A. Anohina, Agents in intelligent tutoring systems: state of the art, Scientific proceedings of Riga Technical University, Computer science, 5th series, Vol. 22, pp. 110-121, 2005.

[12] G. Hume, J. A. Michael, A. A. Rovick, and M. Evens, Hinting as a Tactic in One-on-One Tutoring, The Journal of the Learning Science, Vol. 5, No. 1, pp. 23-47, 1996.

[13] N. Matsuda and K. VanLehn, Modeling Hinting Strategies for Geometry Theorem Proving, Proceedings of 9th International Conference on User Modeling, Johnstown, PA, USA, pp. 373-377, 2003.

[14] Kinshuk, T. Lin, A. Yang and A. Patel, Plug-able Intelligent Tutoring and Authoring: an Integrated Approach to Problem-based Learning, International Journal of Continuing Engineering Education and Life-Long Learning, Vol. 13, No. 1/2, pp. 95-105, 2002.

[15] M. A. Nunes, L. L. Dihl, L. M. Fraga, C. R. Woszezenki, L. Oliveira, D. J. Francisco, G. Machado, C. Nogueira and M. Notargiacomo, IVTE - Pedagogical Game for Distance Learning, Proceedings of ASET Conference, Melbourne, 2002.

[16] P. Suraweera, An Animated Pedagogical Agent for SQL-Tutor, Honours Project HONS 08/99, 1999.

[17] M. Kalayar, H. Ikematsu, T. Hirashima and A. Takeuchi, Intelligent Tutoring System for Search Algorithm, Proceedings of ICCE, Seoul, Korea, pp. 1369-1376, 2001.

[18] M. Stern, J. Beck and B. P. Woolf, Adaptation of Problem Presentation and Feedback in an Intelligent Mathematics Tutor, In C. Frasson, G. Gauthier and A. Lesgold (Eds): Intelligent Tutoring Systems, Springer-Verlag, New York, 1996.

[19] B. A. Cheikes, GIA: An Agent-Based Architecture for Intelligent Tutoring Systems, Proceedings of the CIKM'95 Workshop on Intelligent Information Agents, Baltimore, Maryland, USA, 1995.

[20] N. Capuano, M. De Santo, M. Marsella, M. Molinara and S. Salerno, A Multi-Agent Architecture for Intelligent Tutoring, Proceedings of the International Conference on Advances in Infrastructure for Electronic Business, Science, and Education on the Internet SSGRR 2000, L'Aquila, 2000.

[21] A. M. Bell and S. Ramachandran, An Intelligent Tutoring System for Remote Sensing and Image Interpretation, Proceedings of the Interservice/Industry Training, Simulation, and Education Conference (I/ITSEC), Orlando, Florida, USA, 2003.

[22] C. J. Butz, S. Hua and R. B. Maguire, A Web-based Intelligent Tutoring System for Computer Programming, Proceedings of the IEEE/WIC/ACM International Conference on Web Intelligence (WI'04), Beijing, China, pp. 159-165, 2004. 
[23] E. Remolina, S. Ramachandran, D. Fu, R. Stottler and W. R. Howse, Intelligent Simulation-Based Tutor for Flight Training, Proceedings of the Interservice/Industry Training, Simulation, and Education Conference (I/ITSEC), Orlando, Florida, USA, 2004.

[24] J. M. Gascueña and A. Fernández-Caballero, An Agent-based Intelligent Tutoring System for Enhancing E-learning/ E-teaching, International Journal of Instructional Technology and Distance Learning, Vol. 2, No. 11 , pp. 11-24, 2005.

[25] P. Brusilovsky and C. Peylo, Adaptive and Intelligent Web-based Educational Systems, International Journal of Artificial Intelligence in Education, Vol. 13, pp. 156-169, 2003.

[26] D. R. Benyon and D. M. Murray, Adaptive Systems: from Intelligent Tutoring to Autonomous Agents, Knowledge-Based Systems, Vol. 6, No. 4, pp. 197-219, 1993.

[27] C. Frasson, T. Mengelle and E. Aimeur, Using Pedagogical Agents in a Multi-Strategic Intelligent Tutoring System, Proceedings of the 8th World Conference on Artificial Intelligence in Education AI-ED97, Workshop V: Pedagogical Agents, Kobe, Japan, pp. 40-47, 1997.

[28] A. Anohina, The Problem-Solving Modes and a Two-Layer Model of Hints in the Intelligent Tutoring System for Minimax Algorithm, Proceedings of the 1st International Conference on Virtual Learning, Bucharest, Romania, pp. 105-112, 2006.

[29] G. F. Luger, Artificial Intelligence: Structures and Strategies for Complex Problem Solving, Addison Wesley, 2001.

[30] S. Russell and P. Norvig, Artificial Intelligence: A Modern Approach, Prentice Hall, 2003.

[31] A. Anohina, Intelligent Tutoring System for Minimax Algorithm, Scientific proceedings of Riga Technical University, Computer science, 5th series, Vol. 22, pp. 122-130, 2005.

\author{
Alla Anohina \\ Riga Technical University \\ Department of Systems Theory and Design \\ Kalku street 1, Riga, Latvia, LV-1658 \\ E-mail: alla.anohina@cs.rtu.lv \\ Received: November 8, 2006
}

Editor's note about the author:

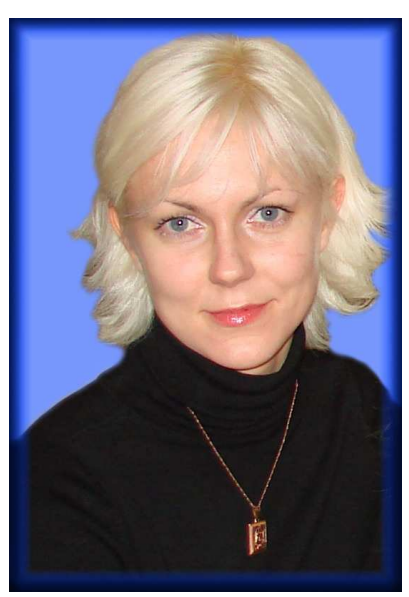

Alla Anohina is an assistant at the Department of System Theory and Design of Riga Technical University in Latvia. She got M.Sc.ing. in 2002 from Riga Technical University and received Werner von Siemens Excellence Award, Award of Latvian Fund of Education and Award and memorial medal of Latvian Academy of Sciences, Lattelecom Ltd. and Latvian Fund of Education for the best master's thesis in the year 2002. Her main research fields are intelligent tutoring systems, computer-assisted assessment systems and artificial intelligence. Now she is finishing her $\mathrm{Ph} . \mathrm{D}$. thesis which main topic is related with the development of the intelligent supporting system for adaptive learning and knowledge assessment. She has five years' experience of teaching in the field of computer science both in Riga Technical University, and in other educational institutions of Latvia. She has participated in several research projects related with the development of knowledge assessment software. 